\title{
Comparação de métodos de análise de adaptabilidade e estabilidade fenotípica em feijoeiro-comum
}

\author{
Helton Santos Pereira( ${ }^{(1)}$, Leonardo Cunha Melo(1), Maria José Del Peloso(1), Luís Cláudio de Faria(1), \\ Joaquim Geraldo Cáprio da Costa( ${ }^{(1)}$, José Luís Cabrera Díaz ${ }^{(1)}$, Carlos Augustín Rava ${ }^{(1)}$ \\ e Adriane Wendland ${ }^{(1)}$
}

${ }^{(1) E m b r a p a ~ A r r o z ~ e ~ F e i j a ̃ o, ~ C a i x a ~ P o s t a l ~ 179, ~ C E P ~ 75375-000 ~ S a n t o ~ A n t o ̂ n i o ~ d e ~ G o i a ́ s, ~ G o . ~ E-m a i l: ~ h e l t o n @ c n p a f . e m b r a p a . b r, ~ l e o n a r d o @ c n p a f . e m b r a p a . b r, ~}$
mjpeloso@cnpaf.embrapa.br, Icfaria@cnpaf.embrapa.br, caprio@cnpaf.embrapa.br, cabrera@cnpaf.embrapa.br, adrianew@cnpaf.embrapa.br

Resumo - O objetivo deste trabalho foi comparar métodos de estudo de adaptabilidade e estabilidade fenotípica de feijoeiro-comum (Phaseolus vulgaris). Foram utilizadas avaliações de produtividade em 71 ensaios, com 16 genótipos em cada um. A adaptabilidade e a estabilidade dos genótipos foi avaliada por meio de seis métodos, e a associação entre os métodos foi determinada pela correlação de Spearman. Os métodos de Cruz e de Eberhart \& Russel apresentaram forte correlação entre si. Os métodos de Lin \& Binns, Lin \& Binns modificado e Annicchiarico também apresentaram alta correlação entre si. Correlações intermediárias foram observadas entre os métodos de Eberhart \& Russel e da análise da interação multiplicativa dos efeitos principais aditivos (AMMI). A utilização conjunta de métodos que apresentaram alta correlação não é indicada. Dessa forma, apenas um dos métodos, entre os de Cruz, Eberhart \& Russel e AMMI, deve ser utilizado em conjunto com Lin \& Binns, Lin \& Binns modificado ou Annicchiarico, visto que não houve correlação entre métodos desses dois grupos. Os métodos de Lin \& Binns modificado e Annicchiarico são indicados para utilização isolada, pois são de utilização simples e permitem a classificação dos ambientes em favoráveis e desfavoráveis e a identificação dos genótipos mais estáveis e adaptados entre os mais produtivos.

Termos para indexação: Phaseolus vulgaris, AMMI, interação genótipos x ambientes.

\section{Comparison of methods for phenotypic adaptability and stability analysis in common bean}

\begin{abstract}
The objective of this work was to compare methods for studying phenotypic adaptability and stability in common beans (Phaseolus vulgaris). Grain yield evaluations were carried out in 71 trials with 16 common bean genotypes, and using six methods. The association among the methods was determined by Spearman correlation. Cruz and Eberhart \& Russel methods were highly correlated. Lin \& Binns, Lin \& Binns modified and Annicchiarico methods were also highly correlated. Intermediate correlations were observed between Eberhart \& Russel and additive main effects and multiplicative interaction model (AMMI) methods. The joint utilization of highly correlated methods is not recommended. Therefore, only one of the methods among Eberhart \& Russel, Cruz and AMMI should be used in association with Lin \& Binns, Lin \& Binns modified or Annicchiarico, since there was no correlation among methods of these two groups. Lin \& Binns and Annicchiarico methods are indicated for isolated utilization, because they are simple to use and allow the classification of environments in favorable and unfavorable, and the identification of the most adapted and stable genotypes among the most productive ones.
\end{abstract}

Index terms: Phaseolus vulgaris, AMMI, genotype x environments interaction.

\section{Introdução}

O feijoeiro-comum é uma cultura de grande importância no Brasil. É cultivado em grande parte do território nacional, submetido às mais variadas condições ambientais e tecnológicas. Para que um programa de melhoramento genético atinja seu objetivo e desenvolva cultivares adaptadas às diversas regiões produtoras do país, é necessário que os genótipos sejam avaliados em um grande número de ambientes, que reflitam as condições ambientais às quais as cultivares serão submetidas.

A ocorrência da interação entre genótipos e ambientes (GxA) tem grande importância, principalmente para o caráter produção de grãos. Uma forma de tirar proveito

Pesq. agropec. bras., Brasília, v.44, n.4, p.374-383, abr. 2009 
dessa interação na indicação de novas cultivares é identificar genótipos com alta adaptabilidade e estabilidade fenotípica.

Vários métodos, baseados em diferentes princípios, foram descritos para avaliação da interação GxA e para a determinação da adaptabilidade e da estabilidade fenotípicas das cultivares. Entre os mais utilizados na cultura do feijoeiro-comum estão aqueles baseados em regressão (Eberhart \& Russel, 1966; Cruz et al., 1989), os não paramétricos (Lin \& Binns, 1988 modificado por Carneiro, 1998; Annicchiarico, 1992) e o método de análise da interação multiplicativa dos efeitos principais aditivos (AMMI) (Gauch \& Zobel, 1996).

$\mathrm{Na}$ escolha dos métodos a serem empregados, devem-se considerar aspectos como facilidade de análise e de interpretação dos resultados (Borges et al., 2000). Oliveira et al. (2006) recomendam a utilização do método de Lin \& Binns (1988) modificado por Carneiro (1998). Já Cargnelutti Filho et al. (2007) indicam que o método de Eberhart \& Russel (1966) deve ser o preferido. A existência de relações entre os métodos também deve ser considerada, principalmente no que se refere ao ordenamento dos genótipos quanto à estabilidade e adaptabilidade. Alguns estudos já foram realizados com o objetivo de comparar os métodos de análise de estabilidade e adaptabilidade. Borges et al. (2000), ao estudar estabilidade fenotípica em feijoeiro-comum, concluíram que o método de Lin \& Binns (1988) ou o de Annicchiarico (1992) devem ser utilizados em conjunto com o método de Toler (1990). Silva \& Duarte (2006), trabalhando com soja, afirmaram que os métodos de Lin \& Binns (1988) e Annicchiarico (1992) não devem ser utilizados em conjunto. Em contrapartida, esses autores recomendam a utilização de um desses métodos em combinação com o de Eberhart \& Russel (1966), assim como a utilização conjunta dos métodos de Eberhart \& Russell (1966) e AMMI. Melo et al. (2007), trabalhando com feijoeirocomum, afirmam que os métodos de Lin \& Binns (1988), Eberhart \& Russel (1966) e AMMI fornecem informações complementares. Silva Filho et al. (2008) recomendam a utilização conjunta dos métodos de Lin \& Binns (1988) e AMMI para estudo de estabilidade fenotípica em algodão.

O programa de melhoramento de feijoeirocomum da Embrapa Arroz e Feijão avalia genótipos promissores, por meio de parcerias, em uma rede nacional de experimentação organizada em ensaios de valor de cultivo e uso (VCU). Esses ensaios são instalados nas épocas de semeadura indicadas em cada região, em municípios de estados que respondem por cerca de $90 \%$ da produção nacional de feijão-comum, durante dois anos. Assim, o conjunto de ensaios obtidos ao final de cada ciclo de VCU (cerca de 80) apresenta grande variação nas condições ambientais, o que possibilita a ocorrência da interação genótipos $\mathrm{x}$ ambientes. Esse número de ensaios é bastante superior ao frequentemente relatado na literatura, em trabalhos que visam à comparação de métodos análise de estabilidade e adaptabilidade, que normalmente usam cerca de 25 ensaios. Portanto, esse conjunto de dados reúne características desejáveis para a realização de um estudo dessa natureza.

O objetivo deste trabalho foi comparar métodos de estudo de adaptabilidade e estabilidade fenotípica de feijoeiro-comum.

\section{Material e Métodos}

Os ensaios foram conduzidos nos anos de 2003 e 2004 em 71 ambientes nos estados de Goiás, Mato Grosso, Mato Grosso do Sul, Tocantins, Paraná, Santa Catarina, São Paulo e no Distrito Federal. O delineamento experimental utilizado foi o de blocos ao acaso, com três repetições e parcelas de quatro linhas com 4 m de comprimento. As duas linhas centrais foram colhidas para determinação da produtividade. Cada ensaio foi constituído por 16 genótipos do grupo carioca (CNFC 9458, CNFC 9471, CNFC 9484, CNFC 9494, CNFC 9500, CNFC 9504, CNFC 9506, CNFC 9518, CNFE 8009, Carioca 11, BRS 9435 Cometa, BRS Estilo, Pérola, Iapar 81, Carioca Pitoco e Magnífico).

Os dados de produtividade foram submetidos à análise de variância, considerando-se o efeito de tratamentos como fixos e os demais como aleatórios. Em seguida, foi realizada a análise conjunta dos ensaios. A razão entre o maior e o menor quadrado médio do resíduo foi superior a sete, evidência de que as variâncias residuais não foram homogêneas (Pimentel-Gomes, 2000). Assim, procedeuse ao ajuste dos graus de liberdade do erro médio e da interação GxA, conforme o método de Cochran (1954). As análises de estabilidade foram realizadas com uso dos métodos de Eberhart \& Russel (1966), Lin \& Binns (1988), Lin \& Binns (1988) modificado por Carneiro (1998), Cruz et al. (1989), Annicchiarico (1992) e AMMI, por meio dos aplicativos GENES (Cruz, 2001) e Estabilidade (Ferreira, 2000). 
No método de Eberhart \& Russell (1966), a adaptabilidade, ou resposta linear aos ambientes, é dada pela estimativa do parâmetro $\beta_{\mathrm{li}}$ e pela produtividade média $\left(\beta_{0 \mathrm{i}}\right)$, enquanto a estabilidade é obtida por meio dos desvios de regressão $\left(\sigma_{\delta i}^{2}\right)$, conforme modelo:

$Y_{i j}=\beta_{0 i}+\beta_{i j} I_{j}+\delta_{i j}+\bar{\varepsilon}_{i j}$, em que: $Y_{i j}$ é a média de produtividade de grãos $\left(\mathrm{kg} \mathrm{ha}^{-1}\right)$ do genótipo i no ambiente j; $\beta_{0 \mathrm{i}}$ é a média geral; $\beta_{\mathrm{li}}$ é o coeficiente de regressão linear; $\delta_{\mathrm{ij}}$ é o desvio da regressão; $\bar{\varepsilon}_{\mathrm{ij}}$ é o erro experimental médio; $\mathrm{I}_{\mathrm{j}}$ é o índice ambiental codificado

$\left(\sum_{j} I_{j}=0\right)$, dado por $I_{j}=\frac{1}{g} \sum_{i} Y_{i j} \frac{1}{a g} Y_{i j}$, para g linhagens e a ambientes.

Na regressão linear bissegmentada de Cruz et al. (1989), a resposta linear aos ambientes desfavoráveis é dada pela estimativa de $\beta_{\mathrm{li}}$, e a resposta a ambientes favoráveis por $\beta_{\mathrm{li}}+\beta_{2 \mathrm{i}}$. A estabilidade dos genótipos é avaliada pelos desvios da regressão $\left(\sigma_{\text {si }}^{2}\right)$ de cada genótipo, em função das variações ambientais. Nesse método, adota-se o seguinte modelo:

$Y_{i j}=\beta_{0 i}+\beta_{l i} I_{i}+\beta_{2 i} T\left(I_{i}\right)+\delta_{i j}+\varepsilon_{i j}$, em que: $Y_{i j}, \beta_{0 i}$, $\mathrm{I}_{\mathrm{j}} \mathrm{e} \bar{\varepsilon}_{\mathrm{ij}}$ são as variáveis definidas anteriormente; e $T\left(I_{j}\right)=0$, se $I_{j}<0$

$\mathrm{T}\left(\mathrm{I}_{\mathrm{j}}\right)=\mathrm{I}_{\mathrm{j}}-\overline{\mathrm{I}}_{+}$, se $\mathrm{I}_{\mathrm{j}}>0$, em que $\overline{\mathrm{I}}_{+}$é a média dos índices $\mathrm{I}_{\mathrm{j}}$ positivos.

O método de Lin \& Binns (1988) foi modificado por Carneiro (1998). A primeira modificação realizada foi a decomposição de $\mathrm{P}_{\mathrm{i}}$ nas partes devidas a ambientes favoráveis e desfavoráveis. Essa primeira modificação será tratada como Lin \& Binns no presente trabalho. A estimativa de $\mathrm{P}_{\mathrm{i}}$ é dada por: $P_{i}=\frac{\sum_{j=1}^{a}\left(Y_{i j}-M_{j}\right)^{2}}{2 a}$, em que: $P_{i}$ é a estimativa

do parâmetro de estabilidade do i-ésimo genótipo; $\mathrm{Y}_{\mathrm{ij}}$ é a produtividade do i-ésimo genótipo no j-ésimo ambiente; $\mathrm{M}_{\mathrm{j}}$ é a resposta máxima observada entre todos os genótipos no j-ésimo ambiente; a é o número de ambientes.

Os ambientes foram classificados com base nos índices ambientais, definidos como a diferença entre a média dos genótipos avaliados em cada local e a média geral. Em ambientes favoráveis, com índices positivos, incluindo o valor 0 , o $\mathrm{P}_{\text {if }}$ foi estimado por equação semelhante à anterior, substituindo-se o termo 2a por $2 \mathrm{f}$, em que f é o número de ambientes favoráveis. Da mesma forma, em ambientes desfavoráveis, cujos índices são negativos, substituiu-se o termo 2a por 2d, em que d é o número de ambientes desfavoráveis.
A segunda modificação realizada por Carneiro (1998) refere-se ao método ponderado pelo coeficiente de variação (CV) (Lin \& Binns modificado). Nesse método, o desempenho de cada genótipo é dado pela estatística a seguir:

$P_{i}=\sum_{j=1}^{n}\left[\left(\frac{Y_{g(j+1)}+Y_{g j}}{2}\right)-\left(\frac{Y_{g(j+1)}+Y_{i j}}{2}\right)\right]^{2}\left(Y_{.(j+1)}-\bar{Y}_{j .}\right)$, em que: $\mathrm{P}_{\mathrm{i}}$ é a estimativa da medida de adaptabilidade e estabilidade de comportamento (MAEC) do genótipo i; $\mathrm{Y}_{\mathrm{ij}}$ é a produtividade do i-ésimo genótipo no j-ésimo ambiente; $\mathrm{Y}_{\mathrm{gj}}$ é a estimativa da produtividade do genótipo hipotético ideal no ambiente j, conforme modelo de Cruz et al. (1989), dado por:

$\mathrm{Y}_{\mathrm{gj}}=\beta_{0 \mathrm{~g}}+\beta_{1 \mathrm{~g}} \mathrm{l}_{\mathrm{j}}+\beta_{2 \mathrm{~g}} \mathrm{~T}\left(\mathrm{l}_{\mathrm{j}}\right)$, em que $\beta_{0 \mathrm{~g}}$ é a máxima produtividade encontrada em todo o ensaio; $\beta_{1 \mathrm{~g}}=0,5$ e $\beta_{2 \mathrm{~g}}=1$ são os valores estabelecidos por Cruz \& Carneiro (2003), um dos quais reflete baixa resposta aos ambientes desfavoráveis $\left(\beta_{1 \mathrm{~g}}=0,5\right)$ e o outro, responsividade a condições favoráveis $\left(\beta_{1 \mathrm{~g}}+\beta_{2 \mathrm{~g}}=1,5\right)$. A estatística $P_{\mathrm{i}}$ é multiplicada pelo fator $\mathrm{F}$, definido por $\mathrm{F}=\mathrm{CV}_{\mathrm{j}} / \mathrm{CV}_{\mathrm{T}}$, em que $C V_{\mathrm{j}}$ é o coeficiente de variação no ambiente $\mathrm{j}$, e $\mathrm{CV}_{\mathrm{T}}$, a soma dos coeficientes de variação dos j ambientes. Assim, linhagens com menor valor de $\mathrm{P}_{\mathrm{i}}$ apresentam comportamento mais próximo ao do genótipo hipotético ideal, além de se levar em consideração a similaridade dos locais, bem como a precisão de cada experimento.

O método de Annicchiarico (1992) é baseado no chamado índice de confiança genotípico, estimado por: $\omega_{i(g)}=\hat{\mu}_{i(g)}-z_{(1-\alpha)} \hat{\sigma}_{z_{i}(g)}$, considerando-se todos os ambientes, em que $\hat{\mu}_{i(g)}$ é a média percentual dos genótipos i em relação à média geral; $\ddot{\sigma}_{z(g)}$ é o desviopadrão dos valores $\mathrm{z}_{\mathrm{ij}}$ associado ao i-ésimo genótipo; $\mathrm{Z}_{(1-\alpha)}$ é o percentil da função de distribuição normal padrão. O índice foi calculado também para os ambientes favoráveis e desfavoráveis. O coeficiente de confiança adotado foi de $75 \%$, isto é, $\alpha=0,25$.

A análise AMMI engloba componentes aditivos, para estudar os efeitos principais, e componentes multiplicativos, para estudar a interação. Na análise de componentes principais, a variação contida nos componentes significativos é chamada de padrão, e a contida nos não significativos, de ruído. Para utilização da metodologia AMMI, o modelo utilizado foi: $Y_{i j}=\mu+g_{i}+a_{j}+\sum_{k=1}^{n} \lambda_{k} \gamma_{i k} \alpha_{j k}+\rho_{i j}+\bar{\varepsilon}_{i j}$, em que: $Y_{i j}$ é a resposta média do genótipo i ( $\mathrm{i}=1,2, \ldots$, G genótipos) 
no ambiente $\mathrm{j}$ ( $\mathrm{j}=1,2, \ldots, \mathrm{A}$ ambientes); $\mu$ é a média geral dos ensaios; $g_{i}$ é o efeito fixo do genótipo $i$; $\mathrm{a}_{\mathrm{j}}$ é o efeito fixo do ambiente $\mathrm{j}$; $\lambda_{\mathrm{k}}$ é o k-ésimo valor singular (escalar) da matriz de interações original (denotada por GA); $\gamma_{\mathrm{ik}}$ é o elemento correspondente ao i-ésimo genótipo no k-ésimo vetor singular coluna da matriz GA; $\alpha_{\mathrm{jk}}$ é o elemento correspondente ao j-ésimo ambiente no k-ésimo vetor singular linha da matriz GA; $\rho_{\mathrm{ij}}$ é o ruído associado ao termo (ga) da interação clássica do genótipo i com o ambiente j; $\bar{\varepsilon}_{\mathrm{ij}}$ é o erro experimental médio. Mais detalhes são apresentados por Duarte \& Vencovsky (1999).

Para a comparação dos métodos, foi estimada a correlação de Spearman entre as ordens de classificação obtidas com base nos parâmetros de estabilidade e adaptabilidade, para cada par de métodos. Para cada método, também foi estabelecida a ordem de classificação quanto à estabilidade e adaptabilidade, utilizando procedimento semelhante ao relatado por Silva \& Duarte (2006), com diferença no ordenamento para $\beta_{\mathrm{li}}$ no método de Eberhart \& Russel e no ordenamento utilizado no método AMMI. Os parâmetros de estabilidade e adaptabilidade fornecidos por cada método foram utilizados com igual peso para definição da classificação dos genótipos.

Para o método de Eberhart \& Russel, foram obtidos os desvios de $\beta_{1 \mathrm{i}}$ em relação a 1 . Os valores absolutos dos desvios foram ordenados e o genótipo com menor valor foi colocado em primeiro lugar. Com relação ao parâmetro $\sigma_{\delta}^{2}$, os genótipos foram ordenados por suas estimativas e o que apresentou menor valor foi colocado em primeiro lugar. Em seguida, foi calculado o posicionamento médio de cada genótipo, obtendose a média das classificações para cada parâmetro. Essas médias foram ordenadas em ordem crescente e os genótipos com menor valor foram os mais estáveis e adaptados.

O mesmo procedimento foi realizado com a metodologia de Cruz et al. para as estimativas de $\sigma_{\delta \dot{x}^{\circ}}^{2}$ Para $\beta_{1 \mathrm{i}}$, as estimativas foram ordenadas em ordem crescente e os genótipos com menor estimativa foram ordenados nos primeiros lugares. Já para o parâmetro $\beta_{1 \mathrm{i}}+\beta_{2 \mathrm{i}}$, o genótipo com a maior estimativa foi ordenado em primeiro lugar. Foi calculado o posicionamento médio dos genótipos para os três parâmetros em conjunto, de modo semelhante ao relatado anteriormente.

Nos métodos de Lin \& Binns e Lin \& Binns modificado, os genótipos foram ordenados pelas estimativas de $\mathrm{P}_{\mathrm{i}}$ para todos os ambientes. O genótipo que apresentou menor estimativa foi considerado o mais estável e adaptado, e assim sucessivamente. Para o método de Annicchiarico, os genótipos foram ordenados pelas estimativas de $I_{i}$ para todos os ambientes, e o de maior estimativa foi classificado como o mais estável e adaptado.

Na metodologia AMMI, foi calculada a média ponderada dos escores absolutos (MPEA) dos dois primeiros componentes principais para cada genótipo, ponderada pela porcentagem da variação explicada por cada componente. Os genótipos foram ordenados pelos valores da MPEA e o de menor média foi considerado mais estável e adaptado.

Como a média de produtividade é muito importante, além da classificação de genótipos descrita anteriormente para as metodologias de Eberhart \& Russel, Cruz e AMMI, também foi obtida uma classificação de genótipos considerando a média de produtividade como um dos parâmetros de estabilidade e adaptabilidade. Nesse caso, os genótipos foram classificados por ordem crescente, a partir da maior média e, posteriormente, obteve-se a classificação dos genótipos considerando todos os parâmetros de cada método, de forma idêntica à relatada anteriormente. Nesse caso, foram utilizados três parâmetros para o método de Eberhart \& Russel (média, $\beta_{1 \mathrm{i}}$ e $\sigma_{\mathrm{si}}^{2}$ ), quatro para Cruz (média, $\beta_{1 \mathrm{i}}, \beta_{1 \mathrm{i}}+\beta_{2 \mathrm{i}}$ e $\sigma_{\delta \mathrm{i}}^{2}$ ) e dois para AMMI (média e MPEA).

\section{Resultados e Discussão}

Houve variação nas médias e CV dos ensaios, o que indica que as condições edafoclimáticas e tecnológicas às quais os genótipos foram submetidos foram discrepantes (Tabela 1). Na análise conjunta, todos os efeitos foram significativos, o que indica a presença de variabilidade entre os genótipos e entre os ambientes utilizados, e também a ocorrência de resposta diferencial dos genótipos aos ambientes, evidência da necessidade de realização de análises de estabilidade (Tabela 2). As estimativas dos parâmetros de adaptabilidade e estabilidade dos genótipos estão apresentadas nas Tabelas 3 e 4.

O método de Cruz et al. somente se correlacionou com o de Eberhart \& Russel, o que indica existir redundância nas informações fornecidas pelos métodos baseados em regressão (Tabela 5). Essa 
Tabela 1. Informações geográficas e resumo das análises de variância individuais dos 71 ensaios de valor de cultivo e uso (VCU) de feijoeiro-comum tipo carioca conduzidos no Brasil nos anos de 2003 e 2004.

\begin{tabular}{|c|c|c|c|c|c|c|c|c|}
\hline Época $^{(1)}$ & Cidade & Estado & Alt. (m) & Lat. S & Long. W & $\mathrm{QME}^{(2)}$ & Média $^{(3)}$ & $\mathrm{CV}$ \\
\hline & & & & & 2003 & & & \\
\hline \multirow[t]{10}{*}{ Seca } & Sto. Ant. Goiás & $\mathrm{GO}$ & 823 & $16^{\circ} 29^{\prime}$ & $49^{\circ} 18^{\prime}$ & 63.884 & 1.275 & 20 \\
\hline & Panamá & $\mathrm{GO}$ & 733 & $18^{\circ} 10^{\prime}$ & $49^{\circ} 21^{\prime}$ & 49.765 & 1.361 & 16 \\
\hline & Dourados & MS & 430 & $22^{\circ} 13^{\prime}$ & $54^{\circ} 48^{\prime}$ & 72.568 & 1.790 & 15 \\
\hline & G. de Dourados & MS & 400 & $22^{\circ} 25^{\prime}$ & $\begin{array}{l}54^{\circ} 13^{\prime} \\
\end{array}$ & 57.563 & 1.426 & 17 \\
\hline & Aquidauana & MS & 147 & $20^{\circ} 28^{\prime}$ & $55^{\circ} 47^{\prime}$ & 26.227 & 1.699 & 10 \\
\hline & Sinop & MT & 345 & $11^{\circ} 51^{\prime}$ & $55^{\circ} 30^{\prime}$ & 65.628 & 1.870 & 14 \\
\hline & Abelardo Luz & $\mathrm{SC}$ & 760 & $26^{\circ} 33^{\prime}$ & $52^{\circ} 19^{\prime}$ & 52.618 & 1.919 & 12 \\
\hline & Ponta Grossa & PR & 969 & $25^{\circ} 05^{\prime}$ & $50^{\circ} 09^{\prime}$ & 144.728 & 2.182 & 17 \\
\hline & Prudentópolis & PR & 840 & $25^{\circ} 12^{\prime}$ & $50^{\circ} 58^{\prime}$ & 107.091 & 2.403 & 14 \\
\hline & Roncador & PR & 762 & $24^{\circ} 36^{\prime}$ & $52^{\circ} 16^{\prime}$ & 44.317 & 1.423 & 15 \\
\hline \multirow[t]{11}{*}{ Inverno } & Planaltina & $\mathrm{DF}$ & 1.000 & $15^{\circ} 27^{\prime}$ & $47^{\circ} 36^{\prime}$ & 91.425 & 2.359 & 13 \\
\hline & Goiatuba & $\mathrm{GO}$ & 447 & $18^{\circ} 00^{\prime}$ & $49^{\circ} 21^{\prime}$ & 53.005 & 1.685 & 14 \\
\hline & Sto. Ant. Goiás & $\mathrm{GO}$ & 823 & $16^{\circ} 29^{\prime}$ & $49^{\circ} 18^{\prime}$ & 86.877 & 1.827 & 16 \\
\hline & Rio Verde & $\mathrm{GO}$ & 715 & $17^{\circ} 47^{\prime}$ & $50^{\circ} 55^{\prime}$ & 117.006 & 2.710 & 13 \\
\hline & Urutaí & $\mathrm{GO}$ & 900 & $17^{\circ} 27^{\prime}$ & $48^{\circ} 12^{\prime}$ & 95.784 & 2.452 & 13 \\
\hline & Cristalina & $\mathrm{GO}$ & 1.239 & $16^{\circ} 46^{\prime}$ & $47^{\circ} 36^{\prime}$ & 569.989 & 3.479 & 22 \\
\hline & Cáceres & MT & 118 & $16^{\circ} 04^{\prime}$ & $57^{\circ} 40^{\prime}$ & 153.598 & 2.478 & 16 \\
\hline & Dueré & TO & 235 & $11^{\circ} 20^{\prime}$ & $49^{\circ} 16^{\prime}$ & 61.740 & $\begin{array}{l}2.4 / 0 \\
1.471\end{array}$ & 17 \\
\hline & Dianópolis & TO & 693 & $11^{\circ} 37^{\prime}$ & $\begin{array}{l}4910 \\
46^{\circ} 49^{\prime}\end{array}$ & 154.771 & 3.347 & 12 \\
\hline & Palmas & TO & 230 & $10^{\circ} 12^{\prime}$ & $48^{\circ} 21^{\prime}$ & 142.905 & 2.258 & 17 \\
\hline & Araguatins & TO & 103 & $05^{\circ} 39^{\prime}$ & $48^{\circ} 07^{\prime}$ & 13.187 & $\begin{array}{r}2.250 \\
836\end{array}$ & 14 \\
\hline \multirow[t]{15}{*}{ Águas } & Planaltina & $\mathrm{DF}$ & 1.000 & $15^{\circ} 27^{\prime}$ & $4736^{\prime}$ & 189.169 & 2.106 & 21 \\
\hline & Rio Verde & GO & 715 & $17^{\circ} 47^{\prime}$ & $50^{\circ} 55^{\prime}$ & 88.291 & 1.891 & 16 \\
\hline & Ipameri & $\mathrm{GO}$ & 605 & $17^{\circ} 43^{\prime}$ & $48^{\circ} 09^{\prime}$ & 127.779 & 2.819 & 13 \\
\hline & Anápolis & $\mathrm{GO}$ & 1.018 & $16^{\circ} 19^{\prime}$ & $48^{\circ} 57^{\prime}$ & 69.003 & 1.369 & 19 \\
\hline & Formosa & $\mathrm{GO}$ & 912 & $15^{\circ} 32^{\prime}$ & $47^{\circ} 20^{\prime}$ & 81.362 & 1.483 & 19 \\
\hline & Sinop & MT & 345 & $11^{\circ} 51^{\prime}$ & $55^{\circ} 30^{\prime}$ & 32.605 & $\begin{array}{l}1.405 \\
973\end{array}$ & 19 \\
\hline & Itapeva & SP & 684 & $23^{\circ} 58^{\prime}$ & $48^{\circ} 52^{\prime}$ & 40.920 & 3.492 & 6 \\
\hline & Capão Bonito & SP & 705 & $24^{\circ} 00^{\prime}$ & $48^{\circ} 20^{\prime}$ & 317.343 & 4.144 & 14 \\
\hline & Taquarituba & SP & 618 & $23^{\circ} 31^{\prime}$ & $49^{\circ} 14^{\prime}$ & 192.663 & 2.289 & 19 \\
\hline & Campos Novos & $\mathrm{SC}$ & 939 & $27^{\circ} 24^{\prime}$ & $51^{\circ} 13^{\prime}$ & 40.713 & 1.190 & 17 \\
\hline & Abelardo Luz & $\mathrm{SC}$ & 760 & $26^{\circ} 33^{\prime}$ & $52^{\circ} 19^{\prime}$ & 346.452 & $\begin{array}{l}1.190 \\
3.028\end{array}$ & 19 \\
\hline & Major Vieira & SC & 786 & $26^{\circ} 22^{\prime}$ & $50^{\circ} 19^{\prime}$ & 128.380 & $\begin{array}{l}3.028 \\
2.120\end{array}$ & 17 \\
\hline & Concórdia & $\mathrm{SC}$ & 569 & $27^{\circ} 14^{\prime}$ & $52^{\circ} 01^{\prime}$ & 120.830 & $\begin{array}{l}2.120 \\
2.440\end{array}$ & 14 \\
\hline & Ponta Grossa & PR & 969 & $25^{\circ} 05^{\prime}$ & $50^{\circ} 09^{\prime}$ & 100.214 & $\begin{array}{l}2.440 \\
3.688\end{array}$ & 9 \\
\hline & Roncador & PR & 762 & $24^{\circ} 36^{\prime}$ & $52^{\circ} 16^{\prime}$ & 35.767 & $\begin{array}{c}J .000 \\
985\end{array}$ & 19 \\
\hline \multirow{9}{*}{ Seca } & & & & & 2004 & & & \\
\hline & Anápolis & GO & 1.018 & $16^{\circ} 19^{\prime}$ & $48^{\circ} 57^{\prime}$ & 91.918 & 1.398 & 22 \\
\hline & Sto. Ant. Goiás & GO & 823 & $16^{\circ} 29^{\prime}$ & $49^{\circ} 18^{\prime}$ & 109.817 & 1.840 & 18 \\
\hline & Aquidauana & MS & 147 & $20^{\circ} 28^{\prime}$ & $\begin{array}{l}49^{\circ} 18 \\
55^{\circ} 47^{\prime}\end{array}$ & 22.022 & 1.297 & 11 \\
\hline & Sinop & MT & 345 & $11^{\circ} 51^{\prime}$ & $\begin{array}{l}55^{\circ} 4 / \\
55^{\circ} 30^{\prime}\end{array}$ & 43.079 & 1.050 & 20 \\
\hline & Abelardo Luz & $\mathrm{SC}$ & 760 & $26^{\circ} 33^{\prime}$ & $\begin{array}{l}5500 \\
52^{\circ} 19^{\prime}\end{array}$ & $\begin{array}{l}43.019 \\
58.524\end{array}$ & 2.331 & 10 \\
\hline & Major Vieira & $\mathrm{SC}$ & 786 & $26^{\circ} 22^{\prime}$ & $\begin{array}{l}5279 \\
50^{\circ} 19^{\prime}\end{array}$ & $\begin{array}{l}50.324 \\
57.126\end{array}$ & 2.067 & 12 \\
\hline & Ponta Grossa & PR & 969 & $25^{\circ} 05^{\prime}$ & $50^{\circ} 09^{\prime}$ & 82.048 & 3.141 & 9 \\
\hline & Prudentópolis & PR & 840 & $25^{\circ} 12^{\prime}$ & $50^{\circ} 58^{\prime}$ & $\begin{array}{r}02.040 \\
136.114\end{array}$ & 2.403 & 15 \\
\hline \multirow[t]{11}{*}{ Inverno } & Planaltina & $\mathrm{DF}$ & 1.000 & $15^{\circ} 27^{\prime}$ & $47^{\circ} 36^{\prime}$ & $\begin{array}{l}130.114 \\
263.357\end{array}$ & 3.853 & 13 \\
\hline & Anápolis & $\mathrm{GO}$ & 1.018 & $16^{\circ} 19^{\prime}$ & $48^{\circ} 57^{\prime}$ & 318.029 & 2.843 & 20 \\
\hline & Sto. AntGoiás & $\mathrm{GO}$ & 823 & $16^{\circ} 29^{\prime}$ & $49^{\circ} 18^{\prime}$ & 139.165 & 2.729 & 14 \\
\hline & Rio Verde & GO & 715 & $17^{\circ} 47^{\prime}$ & $50^{\circ} 55^{\prime}$ & 91.255 & 3.075 & 10 \\
\hline & Itumbiara & $\mathrm{GO}$ & 448 & $18^{\circ} 25^{\prime}$ & $49^{\circ} 12^{\prime}$ & 111.910 & 1.835 & 18 \\
\hline & Morrinhos & $\mathrm{GO}$ & 771 & $17^{\circ} 43^{\prime}$ & $49^{\circ} 05^{\prime}$ & 201.545 & 1.745 & 25 \\
\hline & Cristalina & $\mathrm{GO}$ & 1.239 & $16^{\circ} 46^{\prime}$ & $47^{\circ} 36^{\prime}$ & 311.833 & 2.551 & 22 \\
\hline & Cáceres & MT & 118 & $16^{\circ} 04^{\prime}$ & $\begin{array}{l}4 / 30 \\
57^{\circ} 40^{\prime}\end{array}$ & 68.437 & 2.154 & 12 \\
\hline & Dueré & TO & 235 & $11^{\circ} 20^{\prime}$ & $49^{\circ} 16^{\prime}$ & 76.873 & 1.516 & 18 \\
\hline & Gurupi & TO & 287 & $11^{\circ} 43^{\prime}$ & $49^{\circ} 04^{\prime}$ & 12.735 & 1.437 & 8 \\
\hline & Dianópolis & TO & 693 & $11^{\circ} 37^{\prime}$ & $46^{\circ} 49^{\prime}$ & 139.708 & 2.694 & 14 \\
\hline \multirow[t]{16}{*}{ Águas } & Planaltina & $\mathrm{DF}$ & 1.000 & $15^{\circ} 27^{\prime}$ & $47036^{\prime}$ & $\begin{array}{l}159.100 \\
102.792\end{array}$ & 3.480 & 9 \\
\hline & Anápolis & $\mathrm{GO}$ & 1.018 & $16^{\circ} 19^{\prime}$ & $\begin{array}{l}4 / 30 \\
48^{\circ} 57^{\prime}\end{array}$ & $\begin{array}{l}102.192 \\
107.186\end{array}$ & $\begin{array}{l}5.400 \\
2.307\end{array}$ & 14 \\
\hline & Sto. Ant. Goiás & $\mathrm{GO}$ & 823 & $16^{\circ} 29^{\prime}$ & $\begin{array}{l}48^{\circ} 51 \\
49^{\circ} 18^{\prime}\end{array}$ & $\begin{array}{r}107.180 \\
22.412\end{array}$ & $\begin{array}{l}2.501 \\
1.091\end{array}$ & 14 \\
\hline & Rio Verde & $\mathrm{GO}$ & 715 & $17^{\circ} 47^{\prime}$ & $\begin{array}{l}49^{\circ} 18 \\
50^{\circ} 55^{\prime}\end{array}$ & $\begin{array}{r}22.412 \\
145.613\end{array}$ & $\begin{array}{l}1.091 \\
2.708\end{array}$ & 14 \\
\hline & Morrinhos & $\mathrm{GO}$ & 771 & $17^{\circ} 43^{\prime}$ & $49^{\circ} 05^{\prime}$ & $\begin{array}{r}14 J .015 \\
97.059\end{array}$ & 1.687 & 18 \\
\hline & Urutaí & $\mathrm{GO}$ & 900 & $17^{\circ} 27^{\prime}$ & $48^{\circ} 12^{\prime}$ & 29.728 & 1.755 & 10 \\
\hline & Cristalina & $\mathrm{GO}$ & 1.239 & $16^{\circ} 46^{\prime}$ & $\begin{array}{l}4012 \\
47^{\circ} 36^{\prime}\end{array}$ & 36.981 & 1.286 & 15 \\
\hline & Itaberá & SP & 651 & $23^{\circ} 51^{\prime}$ & $49^{\circ} 08^{\prime}$ & 203.633 & 2.848 & 16 \\
\hline & Paranapanema & SP & 610 & $23^{\circ} 23^{\prime}$ & $48^{\circ} 43^{\prime}$ & 148.305 & 2.556 & 15 \\
\hline & Taquarituba & SP & 618 & $23^{\circ} 31^{\prime}$ & $49^{\circ} 14^{\prime}$ & 110.677 & 2.778 & 12 \\
\hline & Abelardo Luz & $\mathrm{SC}$ & 760 & $26^{\circ} 33^{\prime}$ & $52^{\circ} 19^{\prime}$ & 80.233 & 3.946 & 7 \\
\hline & Major Vieira & SC & 786 & $26^{\circ} 22^{\prime}$ & $50^{\circ} 19^{\prime}$ & 123.320 & 3.012 & 12 \\
\hline & Campos Novos & SC & 939 & $27^{\circ} 24^{\prime}$ & $51^{\circ} 13^{\prime}$ & 48.681 & 1.303 & 17 \\
\hline & Ponta Grossa & PR & 969 & $25^{\circ} 05^{\prime}$ & $50^{\circ} 09^{\prime}$ & 200.558 & 3.202 & 14 \\
\hline & Laranjeir. do Sul & PR & 840 & $25^{\circ} 24^{\prime}$ & $52^{\circ} 24^{\prime}$ & 56.942 & 2.160 & 11 \\
\hline & Londrina & PR & 585 & $23^{\circ} 18^{\prime}$ & $51^{\circ} 09^{\prime}$ & 33.368 & 1.399 & 13 \\
\hline
\end{tabular}

${ }^{(1)}$ Época de semeadura. ${ }^{(2)}$ Quadrado médio do erro. ${ }^{(3)}$ Média geral do ensaio $\left(\mathrm{kg} \mathrm{ha}^{-1}\right) .{ }^{(4)}$ Coeficiente de variação (\%). 
redundância pode ser atribuída à não identificação de genótipo com comportamento ideal segundo o método de Cruz. Nessa situação, esses métodos tendem a fornecer resultados semelhantes. Esses métodos não apresentaram correlações com os demais, exceto Eberhart \& Russel com AMMI, o que reforça a ideia de que métodos baseados em diferentes princípios devem ser utilizados em conjunto (Borges et al., 2000; Silva \& Duarte, 2006).

Os métodos de Lin \& Binns, Lin \& Binns modificado e Annicchiarico apresentaram alta correlação entre si, evidência de que proporcionam informações semelhantes (Tabela 5). A similaridade entre os métodos de Lin \& Binns e Annicchiarico foi relatada por Borges et al. (2000), Silva \& Duarte (2006) e Silva Filho et al. (2008), que atribuíram a semelhança ao fato de que esses métodos avaliam a superioridade dos genótipos, tomando-se como referência os melhores genótipos em cada ambiente (Lin \& Binns e Lin \& Binns modificado) e a média de cada um dos ambientes (Annicchiarico). A correlação entre os métodos de Lin \& Binns e Annicchiarico foi de 0,80 , enquanto que entre Lin \& Binns modificado e Annicchiarico a estimativa foi de 0,94 (Tabela 5), o maior valor encontrado entre os métodos estudados. Isso mostra que as modificações inseridas por Carneiro (trapézio quadrático ponderado pelo CV) causam alteração no ordenamento dos genótipos quando os CV dos experimentos são incluídos no modelo. Entretanto, na prática, os três métodos proporcionaram resultados similares, a julgar pelo fato de que os três genótipos mais estáveis e adaptados identificados por cada método foram os mesmos (BRS Estilo, CNFC 9518 e CNFC 9458), considerando-se todos ambientes.

Tabela 2. Análise de variância conjunta para a produtividade de grãos $\left(\mathrm{kg} \mathrm{ha}^{-1}\right)$ dos 71 ensaios de feijoeiro-comum conduzidos no Brasil, nos anos de 2003 e 2004.

\begin{tabular}{lcc}
\hline Fontes de variação & Graus de liberdade & Quadrado médio \\
\hline Repetição/Ambiente & 142 & $377.780^{* *}$ \\
Ambientes (A) & 70 & $30.853 .051^{* *}$ \\
Genótipos (G) & 15 & $3.326 .020^{* *}$ \\
AxG & $638^{(1)}$ & $659.322^{* *}$ \\
Resíduo & $1.282^{(1)}$ & 187.655 \\
\hline Total & 3.407 & - \\
\hline Média & 2.028 \\
CV (\%) & 21,9 \\
${ }^{(1)}$ Graus de liberdade ajustados (Cochran, 1954). **Significativo a 1\% de \\
probabilidade, pelo teste F.
\end{tabular}

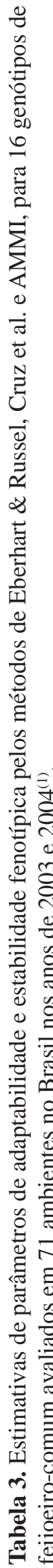




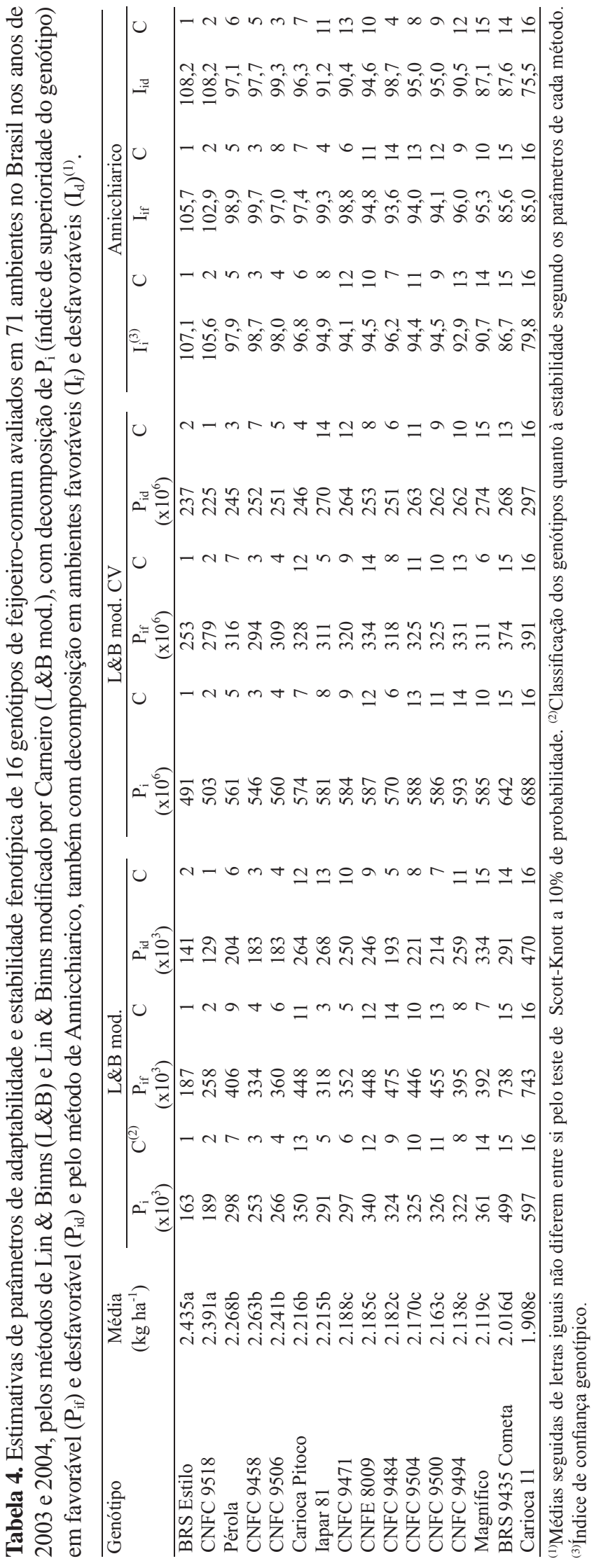

Os métodos de Lin \& Binns modificado e de Annicchiarico não mostraram associação com AMMI, o que indica que um deles pode ser utilizado em conjunto com AMMI. O método de Lin \& Binns apresentou correlação significativa $(0,51)$ com AMMI, indicativo de certo grau de semelhança nas informações obtidas. Melo et al. (2007) também observaram baixa associação entre esses métodos. Silva \& Duarte (2006) não observaram correlação significativa entre eles, evidência de que esses métodos podem ser utilizados em conjunto em algumas situações, conforme sugerido por Silva Filho et al. (2008). Na análise AMMI, os dois primeiros componentes foram significativos, explicaram pequena percentagem da variação total da interação $(17,4$ e $14,2 \%$, respectivamente) e foram utilizados para classificação dos genótipos (Figura 1).

Os métodos de Lin \& Binns, Lin \& Binns modificado e Annicchiarico não apresentaram correlação com Eberhart \& Russel, de forma semelhante aos relatos de Melo et al. (2007) e Silva \& Duarte (2006). Assim, pode-se inferir que a utilização de um desses métodos em conjunto com Eberhart \& Russel pode fornecer informações complementares sobre a estabilidade fenotípica.

Silva \& Duarte (2006) observaram correlação de baixa magnitude entre os métodos de Eberhart \& Russel e AMMI. Esses autores indicaram a utilização conjunta dos dois métodos caso a baixa associação seja confirmada em outras situações, visto que eles podem complementar-se, já que o método de Eberhart \& Russell informa sobre o comportamento de cada genótipo de acordo com a melhoria ambiental e o método AMMI informa sobre a contribuição dos genótipos para a interação genótipos x ambientes. Melo et al. (2007) também encontraram baixa correlação entre esses métodos. No presente trabalho, a correlação entre eles foi intermediária $(0,67)$ e revela um maior grau de associação que os relatados anteriormente, o que, como consequência, contraindica a sua utilização conjunta.

A adoção de novas cultivares depende de vários aspectos. Algumas características das cultivares assumem maior importância nesse contexto, como a produtividade de grãos. Assim, é interessante identificar os genótipos estáveis e adaptados entre os mais produtivos. Entre os métodos avaliados, 
Tabela 5. Estimativas de coeficientes de correlação de Spearman para os parâmetros de adaptabilidade e estabilidade de cada par de métodos e médias ( $\mathrm{kg} \mathrm{ha}^{-1}$ ) obtidas para 16 genótipos em 71 ensaios de feijoeiro-comum no Brasil em 2003 e 2004.

\begin{tabular}{|c|c|c|c|c|c|c|c|c|c|}
\hline Métodos & Média & $\mathrm{E} \& \mathrm{R}$ & Cruz & L\&B & L\&B mod. & Annic. & AMMI & $\mathrm{E} \& \mathrm{R}_{\mathrm{med}}$ & $\mathrm{Cruz}_{\mathrm{med}}$ \\
\hline${\mathrm{E} \& \mathrm{R}^{(1)}}$ & 0,06 & - & & & & & & & \\
\hline $\mathrm{Cruz}^{(2)}$ & 0,09 & $0,75^{*}$ & - & & & & & & \\
\hline $\mathrm{L}^{\prime} \& \mathrm{~B}^{(3)}$ & $0,84 *$ & 0,27 & 0,15 & - & & & & & \\
\hline L\&B mod. ${ }^{(4)}$ & $0,92 *$ & 0,01 & 0,06 & $0,81 *$ & - & & & & \\
\hline Annic. ${ }^{(5)}$ & $0,95 *$ & 0,02 & 0,11 & $0,80 *$ & $0,94 *$ & - & & & \\
\hline $\mathrm{AMMI}^{(6)}$ & 0,23 & $0,67 *$ & 0,43 & $0,51 *$ & 0,26 & 0,21 & - & & \\
\hline$E \& R_{\text {med }}^{(7)}$ & 0,33 & $0,89 *$ & $0,75^{*}$ & $0,58 *$ & 0,35 & 0,38 & $0,68^{*}$ & - & \\
\hline $\mathrm{Cruz}_{\text {med }}{ }^{(7)}$ & 0,28 & $0,69 *$ & $0,90^{*}$ & 0,47 & 0,38 & 0,44 & 0,45 & $0,78^{*}$ & - \\
\hline $\mathrm{AMMI}_{\text {med }}{ }^{(7)}$ & $0,77 *$ & 0,31 & 0,15 & $0,85^{*}$ & $0,72 *$ & $0,69 *$ & $0,76^{*}$ & $0,58^{*}$ & 0,42 \\
\hline
\end{tabular}

${ }^{(1)}$ Eberhart \& Russel (1966). ${ }^{(2)}$ Cruz et al. (1989). ${ }^{(3)}$ Lin \& Binns (1988). (4) Lin \& Binns (1988) modificado por Carneiro (1998). ${ }^{(5)}$ Annicchiarico (1992). ${ }^{(6)}$ Método AMMI. ${ }^{(7)}$ Eberhart \& Russel (1966), Cruz et al. (1989) e AMMI utilizando a média como um dos parâmetros de estabilidade. *Significativo a 5\% de probabilidade, pelo teste t de Student.
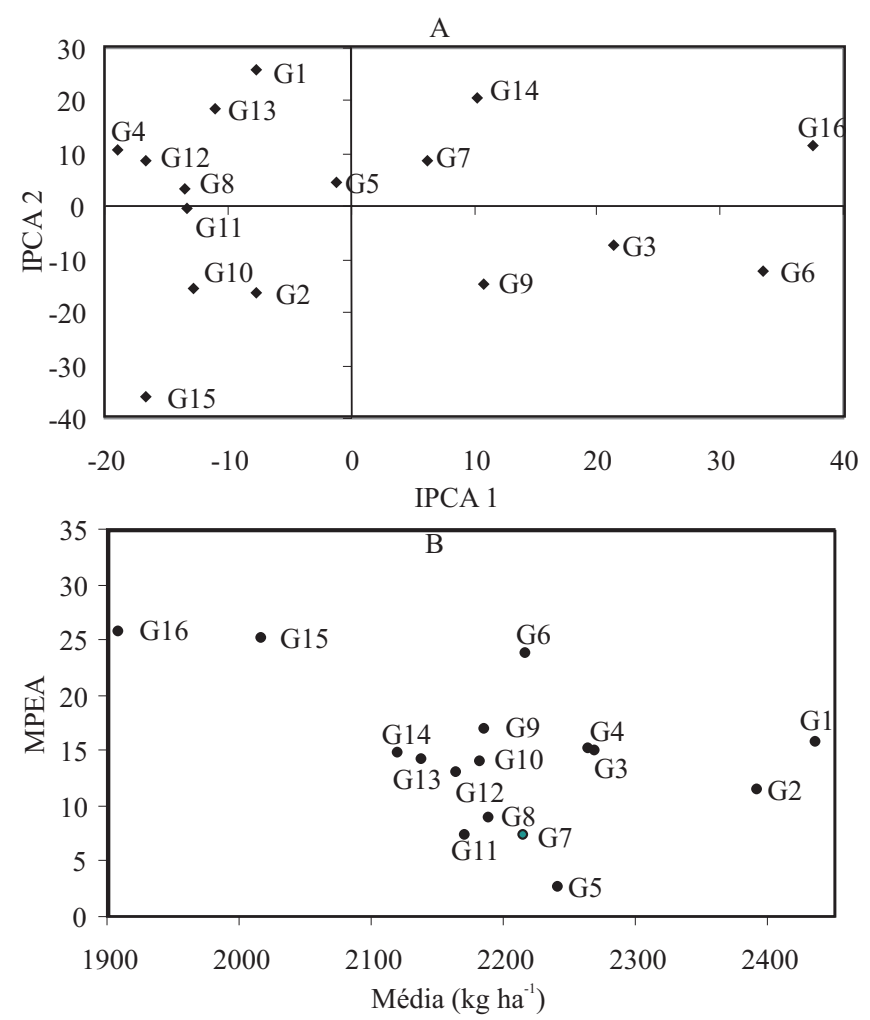

Figura 1. Análises gráficas da análise da interação multiplicativa dos efeitos principais aditivos (AMMI) para os 16 genótipos (G1, BRS Estilo; G2, CNFC 9518; G3, Pérola; G4, CNFC 9458; G5, CNFC 9506; G6, Carioca Pitoco; G7, Iapar 81; G8, CNFC 9471; G9, CNFE 8009; G10, CNFC 9484; G11, CNFC 9504; G12, CNFC 9500; G13, CNFC 9494; G14, Magnífico; G15, BRS 9435 Cometa; G16, Carioca 11), avaliados em 71 ambientes no Brasil. A, primeiro componente principal (IPCA1) $\mathrm{x}$ segundo componente principal (IPCA2); B, média ponderada dos escores absolutos (MPEA) x médias de produtividade $\left(\mathrm{kg} \mathrm{ha}^{-1}\right)$.
Eberhart \& Russel, Cruz e AMMI não apresentaram correlação significativa com a média dos genótipos, o que indica que os genótipos identificados como mais estáveis e adaptados não são, necessariamente, os mais produtivos. Um exemplo disso é que os dois genótipos de maior média de produtividade (BRS Estilo e CNFC 9518) foram classificados, respectivamente, como o $14^{\circ}$ e o $7^{\circ}$ em estabilidade e adaptabilidade pelo método de Eberhart \& Russel, $9^{\circ}$ e $5^{\circ}$ por Cruz e $12^{\circ}$ e $5^{\circ}$ por AMMI (Tabela 3 ). $\mathrm{O}$ método de Eberhart \& Russel identificou os genótipos CNFC 9494 e CNFC 9504 como os mais estáveis e adaptados. Entretanto, eles foram o $13^{\circ}$ e o $11^{\circ} \mathrm{em}$ produtividade, respectivamente. Os genótipos CNFC 9504 e CNFC 9484, identificados como os mais estáveis e adaptados por Cruz, foram o $11^{\circ}$ e o $10^{\circ}$ em produtividade. Já o método AMMI identificou 'CNFC 9506' e 'Iapar 81' como mais estáveis e adaptados, que foram o $5^{\circ}$ e o $7^{\circ}$ em produtividade.

Já os métodos de Lin \& Binns, Lin \& Binns modificado e Annicchiarico mostraram alta correlação com as médias de produtividade (Tabela 5), o que indica que os genótipos identificados como mais estáveis e adaptados por esses métodos deverão figurar entre os mais produtivos. Os três métodos identificaram os genótipos mais produtivos (BRS Estilo e CNFC 9518) como os mais estáveis e adaptados na média de todos os ambientes. Assim, esses métodos têm vantagem prática para utilização, pois, além de identificar os genótipos mais estáveis e adaptados entre os mais produtivos, apresentam simplicidade de aplicação e interpretação do parâmetro que mede a estabilidade e adaptabilidade. Além disso, possuem a vantagem de identificar 
diretamente genótipos adaptados a ambientes favoráveis (com maior média) e desfavoráveis (com menor média).

As correlações obtidas não consideram, para Eberhart \& Russel, Cruz e AMMI, a média de produtividade dos genótipos, embora esse caráter seja de extrema importância no processo de seleção de cultivares. Silva \& Duarte (2006) comentam que a inclusão da média de produtividade possivelmente causaria modificações na classificação dos genótipos e, consequentemente, alteração na correlação entre os métodos.

Com a inclusão da média de produtividade como parâmetro na classificação dos genótipos quanto à estabilidade e adaptabilidade nos métodos de Eberhart \& Russel e Cruz, foi detectado aumento da correlação entre eles e os métodos de Lin \& Binns, Lin \& Binns modificado e Annicchiarico (Tabela 5). Entretanto, a únicaalteração de significância ocorreu com a correlação entre os métodos de Eberhart \& Russel e Lin \& Binns, que passou a ser significativa, porém baixa $(0,58)$, conforme comentado por Silva \& Duarte (2006). Já as correlações de Eberhart \& Russel e Cruz com AMMI praticamente não se alteraram. A inclusão da média no método AMMI causou diminuição da correlação com os métodos de Eberhart \& Russel e Cruz, o que já era esperado, considerando-se a baixa correlação que esses métodos apresentam com a média. As correlações de AMMI com os métodos de Lin \& Binns, Lin \& Binns modificado e Annicchiarico aumentaram e passaram a ser significativas com a inclusão das médias, o que pode ser explicado ao levar-se em conta que a média, utilizada em conjunto com o parâmetro de estabilidade obtido pelo método AMMI, apresentou peso de 50\% na classificação final dos genótipos.

\section{Conclusões}

1. É recomendada a utilização conjunta de métodos de estudo de adaptabilidade e estabilidade, sendo um entre os métodos de Lin \& Binns, Lin \& Binns modificado e Annicchiarico e outro entre os métodos de Eberhart \& Russel, Cruz e AMMI.

2. Os métodos de Lin \& Binns modificado e Annicchiarico são indicados, isoladamente, para uso nos estudos de adaptabilidade e estabilidade fenotípica de cultivares de feijoeiro-comum, pois apresentam simplicidade de utilização e identificam genótipos estáveis e adaptados entre os mais produtivos.

\section{Referências}

ANNICCHIARICO, P. Cultivar adaptation and recommendation from alfalfa trials in Northern Italy. Journal of Genetics and Plant Breeding, v.46, p.269-278, 1992.

BORGES, L.C.; FERREIRA, D.F.; ABREU, A.F.B.; RAMALHO, M.A.P. Emprego de metodologias de avaliação da estabilidade fenotípica na cultura do feijoeiro comum (Phaseolus vulgaris L.). Revista Ceres, v.47, p.89-102, 2000.

CARGNELLUTI FILHO, A.; PERECIN, D.; MELHEIROS, E.B.; GUADAGNIN, J.P. Comparação de métodos de adaptabilidade e estabilidade relacionados à produtividade de grãos de cultivares de milho. Bragantia, v.66, p.571-578, 2007.

CARNEIRO, P.C.S. Novas metodologias de análise da adaptabilidade e estabilidade de comportamento. 1998. 168p. Tese (Doutorado) - Universidade Federal de Viçosa, Viçosa.

COCHRAN, W.G. The combination of estimates from different experiments. Biometrics, v.10, p.101-129, 1954.

CRUZ, C.D. Programa Genes: aplicativo computacional em genética e estatística: versão Windows. Viçosa: UFV, 2001. 648p.

CRUZ, C.D.; CARNEIRO, P.C.S. Modelos biométricos aplicados ao melhoramento genético. Viçosa: UFV, 2003. 579p.

CRUZ, C.D.; TORRES, R.A. de; VENCOVSKY, R. An alternative approach to the stability analysis proposed by Silva and Barreto. Revista Brasileira de Genética, v.12, p.567-580, 1989.

DUARTE, J.B.; VENCOVSKY, R. Interação genótipos x ambientes: uma introdução à análise “AMMI”. Ribeirão Preto: Sociedade Brasileira de Genética, 1999. 60p. (Monografias, 9).

EBERHART, S.A.; RUSSELL, W.A. Stability parameters for comparing varieties. Crop Science, v.6, p.36-40, 1966.

FERREIRA, D.F. Aplicativo Estabilidade. Lavras: Ufla, 2000. Disponível em: < www.ufla.br/dex>. Acesso em: 5 maio 2008.

GAUCH, H.G.; ZOBEL, R.W. AMMI analysis of yield trials. In: KANG, M.S.; GAUCH, H.G. (Ed.). Genotype by environment interaction. Boca Raton: CRC Press, 1996. v.4, p.85-122.

LIN, C.S.; BINNS, M.R. A superiority measure of cultivar performance for cultivar $\mathrm{x}$ location data. Canadian Journal Plant Science, v.68, p.193-198, 1988.

MELO, L.C.; MELO, P.G.; FARIA, L.C. de; DIAZ, J.L.C; DEL PELOSO, M.J.; RAVA, C.A.; COSTA, J.G.C. da. Interação com ambientes e estabilidade de genótipos de feijoeiro-comum na Região Centro-Sul do Brasil. Pesquisa Agropecuária Brasileira, v.42, p.715-723, 2007.

OLIVEIRA, G.V.; CARNEIRO, P.C.S.; CARNEIRO, J.E. de S.; CRUZ, C.D. Adaptabilidade e estabilidade de linhagens de feijão comum em 
Minas Gerais. Pesquisa Agropecuária Brasileira, v.41, p.257-265, 2006.

PIMENTEL-GOMES, F.P. Curso de estatística experimental. 11.ed. São Paulo: Nobel, 2000. 466p.

SILVA, W.C.J. e; DUARTE, J.B. Métodos estatísticos para estudo de adaptabilidade estabilidade fenotípica em soja. PesquisaAgropecuária Brasileira, v.41, p.23-30, 2006.
SILVAFILHO, J.L. da; MORELLO, C. de L.; FARIAS, F.J.C.; LAMAS, F.M.; PEDROSA, M.B.; RIBEIRO, J.L. Comparação de métodos para avaliar a adaptabilidade e estabilidade produtiva em algodoeiro. Pesquisa Agropecuária Brasileira, v.43, p.349-355, 2008.

TOLER, J.E. Patterns of genotype performance over environmental arrays. 1990. 154p. Thesis (Ph.D.) - Clemson University, South Carolina, USA.

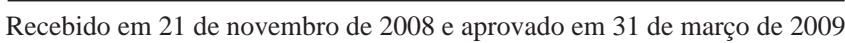

